\title{
Erratum: Causal compatibility inequalities admitting quantum violations in the triangle structure [Phys. Rev. A 98, 022113 (2018)]
}

\author{
Thomas C. Fraser $\odot$ and Elie Wolfe
}

(Received 14 July 2020; published 3 August 2020)

DOI: 10.1103/PhysRevA.102.029901

Our article claimed that inequality (12), reproduced here,

$$
\begin{aligned}
& I_{\text {wagon wheel }}: \\
& \quad+P_{A_{l} B_{l}}(11)-P_{A_{l} B_{l} C_{l} C_{r}}(1111)+P_{A_{l} B_{l}}(00) P_{C_{l} C_{r}}(11)+P_{C_{l} C_{r}}(01) P_{C_{l} C_{r}}(10) \\
& \quad-P_{C_{l} C_{r}}(11) P_{A_{l} A_{r} B_{l} B_{r} C_{l} C_{r}}(000000)-P_{C_{l} C_{r}}(11) P_{A_{l} A_{r} B_{l} B_{r} C_{l} C_{r}}(010100) \\
& \quad-P_{C_{l} C_{r}}(10) P_{A_{l} A_{r} B_{l} B_{r} C_{l} C_{r}}(001001)-P_{C_{l} C_{r}}(10) P_{A_{l} A_{r} B_{l} B_{r} C_{l} C_{r}}(011101) \\
& \quad-P_{C_{l} C_{r}}(01) P_{A_{l} A_{r} B_{l} B_{r} C_{l} C_{r}}(100110)-P_{C_{l} C_{r}}(01) P_{A_{l} A_{r} B_{l} B_{r} C_{l} C_{r}}(110010) \\
& \quad+P_{C_{l} C_{r}}(00) P_{A_{l} A_{r} B_{l} B_{r} C_{l} C_{r}}(101111)+P_{C_{l} C_{r}}(00) P_{A_{l} A_{r} B_{l} B_{r} C_{l} C_{r}}(111011) \\
& \leqslant
\end{aligned}
$$

is a valid causal compatibility inequality for the triangle structure. Additionally, we claimed that the distribution in Eq. (8), namely, Fritz's distrubtion $P_{\mathrm{F}}$, achieves a violation of the above inequality with a numerical value of $\frac{1}{16} \not 0$. Both of these reported claims are false.

One avenue for correcting the above inequality would be to simply reverse the direction of the inequality sign $(\leqslant \mapsto \geqslant)$, or, equivalently, flip the sign of each of the coefficients. Although the inequality resulting from such a correction would, indeed, be a valid causal compatibility inequality for the triangle structure, it has the undesirable feature of being satisfied by Fritz's distribution $P_{\mathrm{F}}$ with a numerical value of $\frac{1}{16} \geqslant 0$.

Therefore, it is our recommendation to replace the incorrect inequality above with the following inequality:

$$
\begin{aligned}
& I_{\text {wagon wheel }}: \\
& \quad-P_{A_{l} B_{l}}(10)+P_{A_{l} B_{l} C_{l} C_{r}}(1010)-P_{A_{l} B_{l}}(01) P_{C_{l} C_{r}}(10)-P_{C_{l} C_{r}}(00) P_{C_{l} C_{r}}(11) \\
& \quad-P_{C_{l} C_{r}}(01) P_{A_{l} A_{r} B_{l} B_{r} C_{l} C_{r}}(100110)-P_{C_{l} C_{r}}(01) P_{A_{l} A_{r} B_{l} B_{r} C_{l} C_{r}}(110010) \\
& \quad+P_{C_{l} C_{r}}(00) P_{A_{l} A_{r} B_{l} B_{r} C_{l} C_{r}}(10111)+P_{C_{l} C_{r}}(00) P_{A_{l} A_{r} B_{l} B_{r} C_{l} C_{r}}(111011) \\
& \quad+P_{C_{l} C_{r}}(10) P_{A_{l} A_{r} B_{l} B_{r} C_{l} C_{r}}(001001)+P_{C_{l} C_{r}}(10) P_{A_{l} A_{r} B_{l} B_{r} C_{l} C_{r}}(011101) \\
& \quad+P_{C_{l} C_{r}}(11) P_{A_{l} A_{r} B_{l} B_{r} C_{l} C_{r}}(000000)+P_{C_{l} C_{r}}(11) P_{A_{l} A_{r} B_{l} B_{r} C_{l} C_{r}}(010100) \\
& \leqslant \\
& 0 .
\end{aligned}
$$

Note that inequality (2) is related to inequality (1) by: (i) flipping the signs of every coefficient, and (ii) flipping the bit values for both $B_{r}$ and $C_{l}$. This substitute inequality is, indeed, a valid causal compatibility inequality for the triangle structure and, moreover, is violated by the Fritz distribution with a numerical violation of $\frac{1}{16}(\sqrt{2}-1) \not 0$.

It is also worth noting that the qualitative features of the wagon-wheel inequality discussed in the "Numerical optimization results" section of the published article were actually determined using, and, thus, pertain to, our proposed substitute inequality (2) not the incorrectly published inequality (1). Consequently, those comments do not require any alteration. Inequality (1) was published in place of inequality (2) due to an error made by the first author during the final drafting stages of the paper.

The diagram appearing in Fig. 1 of our article is incorrectly a duplicate of the diagram appearing in Fig. 3. Instead, Fig. 1 should have been been the following: 


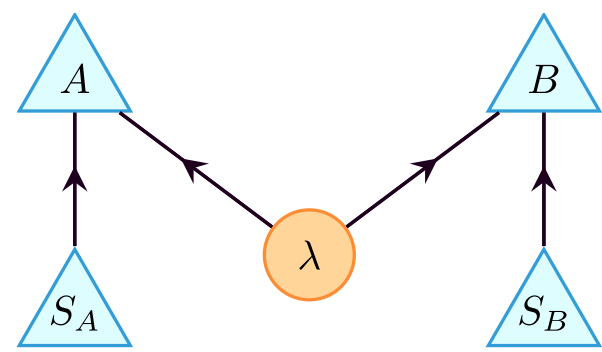

FIG. 1. The Bell structure consisting of two observers $A, B$ together with measurement settings $S_{A}$ and $S_{B}$, respectively. The shared latent variable is labeled $\lambda$.

The authors would like to thank R. Chaves for identifying the error with the wagon-wheel inequality found in earlier versions of this paper. 